\title{
Novel compound heterozygous variants in XYLT1 gene caused Desbuquois dysplasia type 2 in an aborted fetus: a case report
}

\author{
Fatemeh Rajabi ${ }^{1}$, Ali Hosseini Bereshneh², Mahboubeh Ramezanzadeh ${ }^{3}$ and Masoud Garshasbi ${ }^{* *}$
}

\begin{abstract}
Background: Desbuquois dysplasia type 2 (DBQD2) is an infrequent dysplasia with a wide range of symptoms, including facial deformities, growth retardation and short long bones. It is an autosomal recessive disorder caused by mutations in the XYLT1 gene that encodes xylosyltransferase-1.

Case presentation: We studied an aborted fetus from Iranian non-consanguineous parents who was therapeutically aborted at 19 weeks of gestation. Ultrasound examinations at 18 weeks of gestation revealed growth retardation in her long bones and some facial problems. Whole-exome sequencing was performed on the aborted fetus which revealed compound heterozygous XYLT1 mutations: C.742G>A; p.(Glu248Lys) and c.1537 C>A; p.(Leu513Met). Sanger sequencing and segregation analysis confirmed the compound heterozygosity of these variants in XYLT1.

Conclusion: The c.1537 C>A; p.(Leu513Met) variant has not been reported in any databases so far and therefore is novel. This is the third compound heterozygote report in XYLT1 and further supports the high heterogeneity of this disease.
\end{abstract}

Keywords: Desbuquois dysplasia 2, XYLT1 mutations, Skeletal dysplasia, Compound heterozygous

\section{Background}

Desbuquois dysplasia (DBQD; MIM 615,777) is an autosomal recessive skeletal disorder categorized in a group of dysplasia with multiple joint dislocations. DBQD is a heterogeneous condition that overlaps with other skeletal dysplasia [1,2]. The clinical manifestations contain severe prenatal and postnatal growth retardation, frailty joint, round face, midface hypoplasia, prominent eyes, short extremities and progressive scoliosis [3]. According to the presence or absence of hand abnormality, this condition divides into two subfamilies including DBQD1 and DBQD2.

\footnotetext{
*Correspondence: masoud.garshasbi@modares.ac.ir

1 Department of Medical Genetics, Faculty of Medical Sciences, Tarbiat

Modares University, Tehran, Iran

Full list of author information is available at the end of the article
}

In DBQD2, no hand abnormality is observed and it is caused by mutations in the xylosyltransferase 1 gene (XYLT1; MIM 608,124). The DBQD1 is caused by CANT1 (MIM 613,165) mutations. Some forms of DBQD2 are also caused by mutations in CANT1 $[2,4,5]$. XYLT1 gene (NM_022166.4) is located on chromosome $16 \mathrm{p} 12.3$ and consists of 12 exons. This gene encodes xylosyltransferase 1 (XT1) (EC 2.4.2.26, NP_071449.1), which is involved in the proteoglycan (PG) synthesis. PG's structure is made of a core protein with one or more glycosaminoglycan (GAG) chains and XT1 or XT2 transfers xylose from the uridine diphosphate (UDP)-xylose to a specific serine residue of the core protein. [6, 7]. PGs are one of the main parts of the extracellular matrix (ECM). ECM is part of the cell structure and has various biological functions like cellular differentiation and cell growth. Thus, loss of XT protein causes some skeletal dysplasias like osteoarthritis [8]. 
In this study, we applied whole-exome sequencing (WES) on an aborted fetus, who showed skeletal dysplasia in ultrasound at 18 weeks of gestation, which led to the identification of compound heterozygous variants in XYLT1.

\section{Case presentation}

An aborted female fetus around 19 weeks of gestation was referred to the DeNA laboratory, Tehran, Iran. It was the first product of conception for a healthy, Iranian non-consanguineous couple; a 34-year-old mother and a 37-year-old father. Ultrasound at 11 weeks and 6 days of gestation $(C R L=55 \mathrm{~mm})$ reported normal volume of amniotic fluid, nuchal translucency (NT=1.35 $\mathrm{mm})$ and fetal heart rate $(\mathrm{FHR}=171 \mathrm{bmp})$. The fetus was suspected of dysplasia upon routine prenatal ultrasound evaluation performed at 18 gestation weeks. Ultrasound examination at 18 weeks and 3 days of gestation showed that growth of the femur in the fetus was proportional to $<$ 3rd centile (Z- score: -2.53 ) and represented 10 days to 2 weeks delay (Table 1 ). Moreover, the growth in other bones was equivalent to $<3$-10th centile, and one-week growth retardation was seen in the tibia, humerus and ulna bones (Table 1). Her head circumference (HC) was $156 \mathrm{~mm}$ (63th centile) and her abdominal circumference (AC) was equal to $132.7 \mathrm{~mm}$ (56th centile). In addition, the proband had facial characteristics such as frontal bossing, nasal bridge depression, slight midface hypoplasia, small nasal bone (25th centile) and slight brachycephalic. The other biometric parameters including weight (225 gr; 50th centile), amniotic fluid index $(7 \mathrm{~cm})$ and nuchal fold sickness $(2 \mathrm{~mm})$ were normal. Based on the sonographic findings the fetus was therapeutically aborted.

Whole-exome sequencing was performed on the affected fetus using the Nextera Rapid Capture Exome kit which consists of more than 340,000 unique probes to cover 214,405 exons of all human genome chromosomes. The generated library was sequenced on a HiSeq 4000, Illumina (Illumina, Inc., San Diego, CA, USA) with an average coverage depth of $111 \mathrm{X}$. All disease-causing variants reported in $\mathrm{HGMD}^{\circledR}$ and ClinVar as well as the variants with minor allele frequency [MAF] of less than $1 \%$ in the ExAc database were considered. The evaluation was focused on exons and intron boundaries \pm 20 . Burrows-Wheeler Aligner software (version 0.7.15r1140), Genome Analysis Toolkit [GATK] were used for aligning the reads to the reference genome GRCh37 [hg19]. Variant calling, annotation and filtering were performed as previously described [9] and revealed two previously unreported compound heterozygous XYLT1 variants: c.742G $>$ A; p.(Glu248Lys); Chr16 (GRCh37): g.17,353,016 C>T and c.1537 C>A; p.(Leu513Met); Chr16 (GRCh37): g.17235060G $>\mathrm{T}$ in the fetus. The c.1537 C>A variant has not been reported in any available databases and was classified as a variant with uncertain significance (class 3) according to the ACMG recommendations. However, the c.742G>A variant (rs765052371) had been reported previously with the allele frequency 0.00009 (ALFA Project). In order to confirm these two missense point mutations in the fetus, Sanger sequencing was performed. In this method, the fragments were amplified using PCR and then PCR products were sequenced by ABI 3130XL capillary electrophoresis. The primers were 5'-GGATGTCTGGGTGTGTAGAGG-3' and 5'-CAG GTTCATTCGGAAGCAGG-3' (for the c.1537 C>A); and $5^{\prime}$ - GAGCAGAATGGGGCTGGG- ${ }^{\prime}$ and $5^{\prime}$ - GGT GAGGTGCTGCCTCC-3' (for the c.742G>A).

There was no history of DBQD2 in her family and relatives. Segregation analysis showed that the mother was heterozygous for the c.742 G>A and the father was heterozygous for the c.1537 C>A variant. Hence, this result confirmed compound heterozygosity of the c.742 G>A and c.1537 C>A variants in the XYLT1 gene (Fig. 1). In order to predict the pathogenicity of the variants, in silico prediction of the functional effect of these alternations at the amino-acid levels was done by PolyPhen-2 (http://geneics.bwh.harvard.edu/pph2), SIFT (http://sift.jcvi.org), PMut (http://mmb.irbbarcelo na.org/PMut), PROVEAN (http://provean.jcvi.org/ index.php) and CADD (https://cadd.gs.washington. edu/) (Supplementary Table 1). XT1 encodes 959 amino acids in the precursor structure that is composed of two domains including the glycosyltransferase family 14 (Core-2/I-Branching enzyme, amino acid 328-581) and xylosyltransferase (Xylo-C, amino acid 613-794) (Fig. 2a). The overall amino acid conservation of XT1 were shown in Fig. 2b. To be more specific, analyzing

Table 1 Fetal body parameters in 18 weeks +5 days of gestation by ultrasound

\begin{tabular}{|c|c|c|c|c|c|c|c|c|}
\hline Fibula length & $\mathrm{TL}$ & $\mathrm{RL}$ & UL & $\mathrm{HL}$ & BPD & $\mathrm{HC}$ & $A C$ & $\mathrm{FL}$ \\
\hline $20.5 \mathrm{~mm}$ & $21 \mathrm{~mm}$ & $21 \mathrm{~mm}$ & $21 \mathrm{~mm}$ & 23 mm & $41 \mathrm{~mm}$ & $150 \mathrm{~mm}$ & $132 . \mathrm{mm}$ & $22 \mathrm{~mm}$ \\
\hline Z-score: & Z-score: & Z-score: & Z-score: & Z-score: & Z-score: & Z-score: & Z-score: & Z-score: \\
\hline-1.47 & -1.47 & -0.92 & -1.94 & & & & +0.34 & -2.51 \\
\hline
\end{tabular}




\section{Genomic DNA \\ ATA} Amino Acid (Normal)

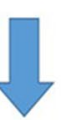

Genomic DNA
Amino Acid Amino Acid
(Mutant)

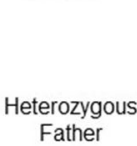
Father

\section{ATA CTT GGT CTT GGG GGA GCT

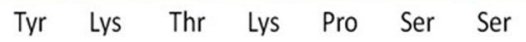

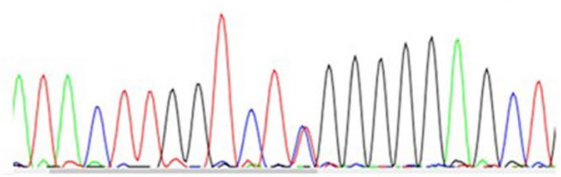

Heterozygous Mother

Genomic DNA Amino Acid (Mutant)

Genomic DNA Amino Acid (Normal)

\section{ATA CTT GGT CTC GGG GGA GCT \\ Tyr Lys Thr Glu Pro Ser Ser}
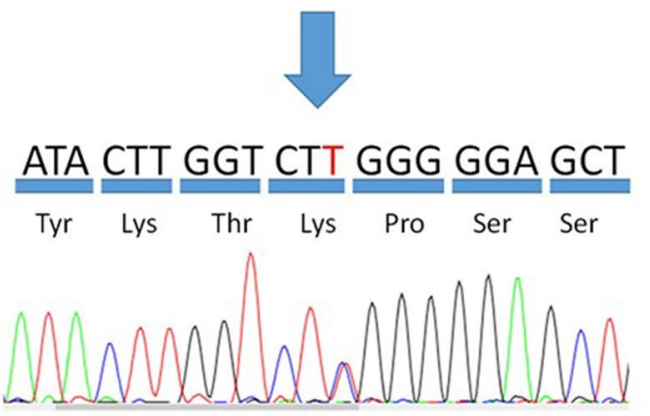

Fig. 1 Sanger sequencing and the mutation segregation

(A)

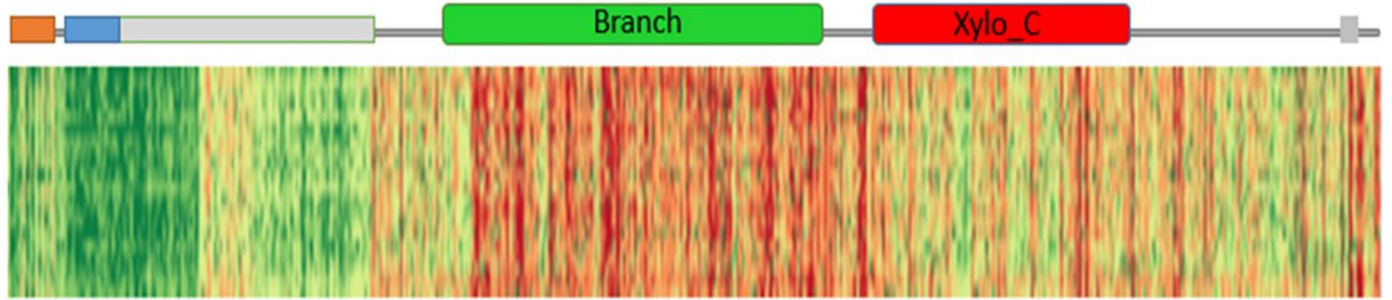

Position score

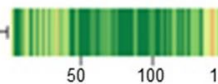

(C)
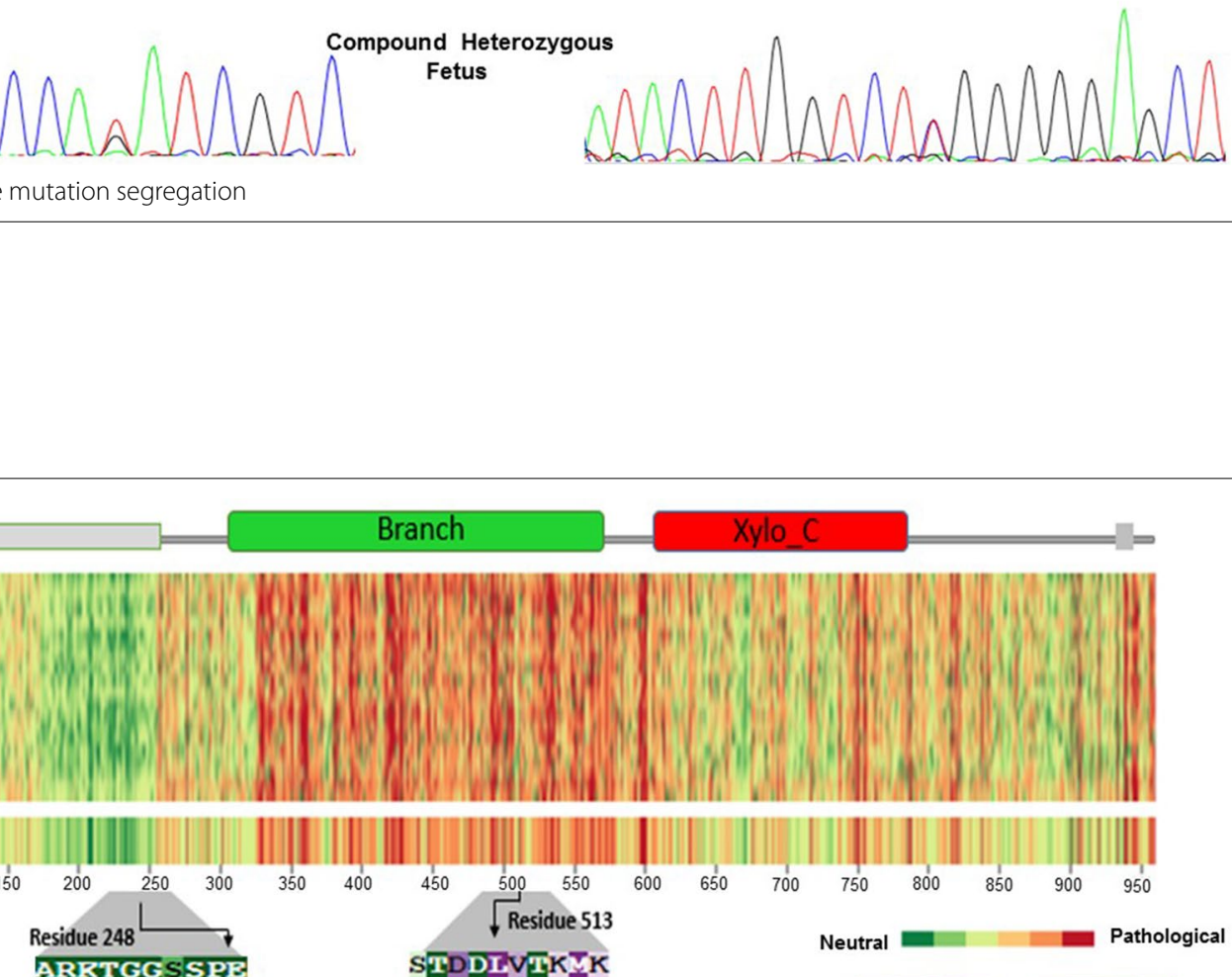

(B)

ARRTGGSSPE

STDDLVTK 프

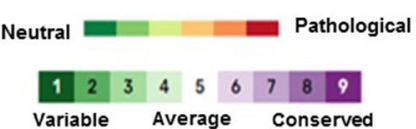

$\begin{array}{lll}\text { Species } & \text { Residue 248 } & \text { Residue 513 } \\ \text { Human } & \text { DYKTEPSSG } & \text { MKTV DDTS } \\ \text { Rhesus } & \text { DYKTEPSSG } & \text { MKTV DDTS } \\ \text { Mouse } & \text { DSKTEPSGG } & \text { MKTV DDTS } \\ \text { Dog } & \text { DYRLEPSGG } & \text { MKTVLDDTS } \\ \text { Elephant } & \text { DYKIEPSSD } & \text { MKTVLDDTS } \\ \text { Chicken } & \text { DYKLEPSSN } & \text { MKTVLDDTK }\end{array}$

Fig. 2 a lllustrates the important domains of XT1 protein. $\mathbf{b}$ The overall view of the protein and conserved domains are illustrated. $\mathbf{c}$ Conservation scores of the residue 513 of the xylosyltransferase 1 protein analyzed by Consurf server. $\mathbf{d}$ Conservation of residues 513 and 248 in vertebrates 


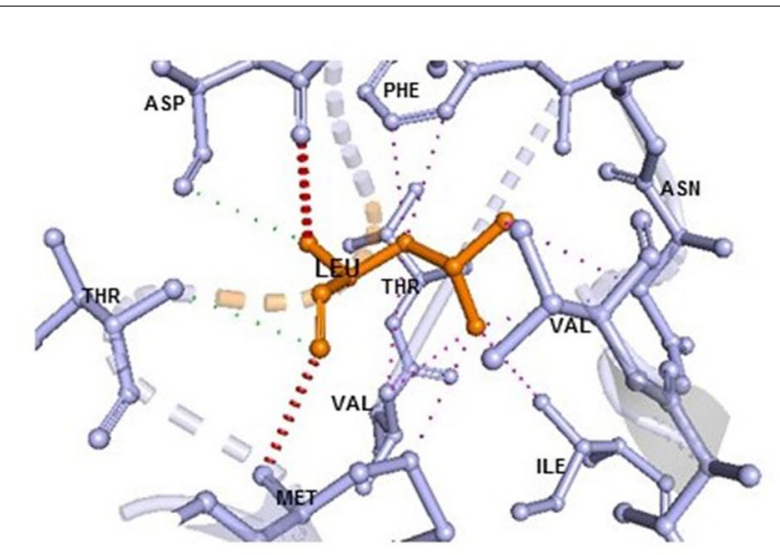

(A)

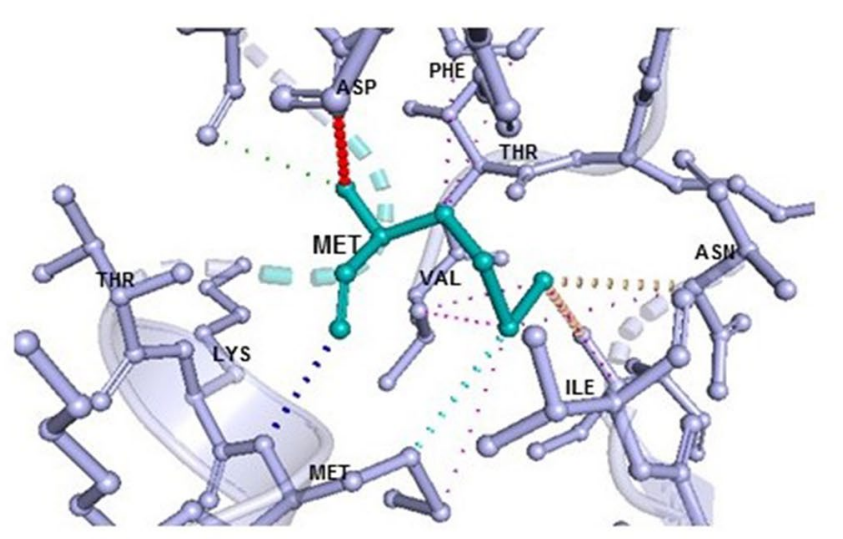

(B)

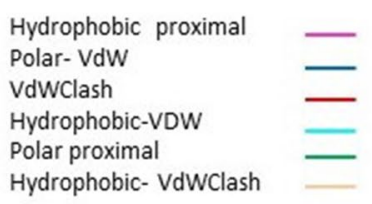

Fig. 3 Effect of p.(Leu513Met) mutation on interatomic interactions of XT1 protein. a Normal; b Mutant. Structural topology has been changed in the mutant form

the conservation of Glu248 and Met513, using ConSurf server (https://consurf.tau.ac.il/), illustrated that Glu248 is not highly conserved unlike Met513 (Fig. 2c). However, amino acid alignment using the UCSC database showed that the 248 and 513 residues are highly conserved in vertebrates (Fig. 2d). E248K alternation is placed in the region of "disorder" and can affect the surrounding amino acids of glutamic acid, including the amino acids serine and threonine. It is predicted that these amino acids are the casein kinase II phosphorylation site and the E248K variant changes this function using different tools such as ScanProsite (https://prosi te.expasy.org/scanprosite/). The L513M variant changes a residue inside the glycosyltransferase domain. Most of the reported mutations in this region lead to reduction or loss of enzyme activity according to UniProtKB (https://www.uniprot.org/). Thus, the L513M variant is supposed to reduce the enzyme activity in this case too. The impact of the novel variant on the protein was depicted by using PyMOL [10], Dynamut [11], OCTOPUS [12] and FOLDING RaCe [13]. Study of L513M in the XT1 protein showed this variant does not influence its hydrogenous interactions, even though it affects other interatomic interactions (Fig. 3) and destabilizes $(\Delta \Delta \mathrm{G}:-0.311 \mathrm{kcal} / \mathrm{mol})$ the protein. Evaluation of L513M showed that the logarithmic change in the folding rate of the protein is equal to $-0.41 / \mathrm{s}$. Moreover, the vibrational entropy energy differences between wild-type and mutant $\left(\Delta \Delta \mathrm{S}_{\mathrm{Vib}} \mathrm{ENCoM}=-4.189 \mathrm{kcal}\right.$. mol-1.K-1) depicted decreased molecule flexibility.

\section{Discussion and conclusion}

The aborted fetuses at 18 weeks of gestation showed short limb bones and facial bone abnormalities on ultrasound. To identify pathogenic variants, we carried out WES, allowing us to identify the genetic cause of DBQD2. Flat face, narrow thorax, low nasal bridge, joint dislocations, Swedish key/monkey wrench appearance, brachymetacarpia and short long bones are the prominent features of the DBQD2 which are mainly observed after birth [14, 15]. Despite this, most cases of DBQD2 show nasal bridge depression and short extremities in ultrasonography during gestational age $[7,16]$. The patient in our study indicated relative macrocephaly (HP: 0004482), depressed nasal bridge (HP: 0005280) and short long bone (HP: 0003026) according to Human Phenotype Ontology terms [17] which are comparable with previous reported cases. Molecular studies of the fetus and her parents revealed unreported compound heterozygous variants in XYLT1 gene. Although various distinct homozygous mutations have been reported in XYLT1, only a few compound heterozygous mutations (c.595 C>T \& c.1651 C>T [1] in a Polish patient and c.1588-10_1595del \& 3.3 Mb del [6] in a Dutch patient) have been reported. In the Dutch patient who had a compound heterozygote mutations in XYLT1 gene, the clinical phenotypes that 
were observed included only short limbs, cleft palate, a short nose with depressed nasal bridge and respiratory problems, without other physical characteristics of DBQD2 [6]. It may indicate that the clinical phenotypes of compound heterozygote mutations are somewhat different from homozygous mutations.

Although various studies have reported different prenatal and postnatal symptoms such as endocrinological problems, in our study, the abortion occurred at 19 weeks which made it impossible to follow the other clinical presentations after birth.

The study by Ranza et al. found that the number of patients with clinical symptoms of DBQD2 had no mutation in the XYLT1 gene. It reveals that DBQD2 has clinical manifestations that overlap with other different skeletal disorders including Larsen syndrome (MIM 150,250, 245,600, LRS), Spondylo-Epi-Metaphyseal Dysplasia with Joint Laxity, leptodactylic type (MIM 603,546, SEMDJL2), Desbuquois dysplasia type 1 and Kim variant, Spondylo-Epiphyseal Dysplasia with dislocations (MIM 143,095) and chondrodysplasia that their pathogenic effects attribute to synthesis or sulfation of proteoglycans (PG) [14]. Another possible explanation could be that DSBQ2 is a heterogeneous disease. Therefore, exome sequencing helps to detect the cause of diseases in cases in which the clinical phenotype indicates one or more syndromes [18].

XT1 and XT2 are type II transmembrane proteins that are included a short amino-terminal region facing the cytosol, a single transmembrane helix and a stem region required for Golgi localization [19]. The variants identified in this study affect amino acid numbers 248 and 513; both of them are part of the luminal topological domain. Mutations in XYLT1 gene could change the topology and structure of protein and therefore not properly localize into the cell membrane; thus, the transferase activity of the XT1 protein will be disrupted.

This enzyme catalyzes the first step in the biosynthesis of PGs like chondroitin sulfate (CS), dermatan sulfate (DS) and heparan sulfate (HS) proteoglycans [20]. The importance of PGs is in cellular homeostasis and impacting many fundamental biological processes including growth factor function, morphogen gradient formation, co-receptor activity, neuronal regeneration, signaling and development of many tissues in humans [21, 22]. Mizumoto et al. in 2015 showed that the biosynthesis of highmolecular-weight CS-PGs, but not HS-PGs, was less in the cells with XYLT1 mutations than in healthy controls because these manifestations may be caused by reductions in CS side chains. These findings indicate that XT1 mainly acts on serine residues in the core proteins of CS-PG, but not HS-PG, and the functions of XYLT1 cannot be compensated by XYLT2 [23].
In conclusion, we report a new DBQD2 case carrying two novel compound heterozygous mutations, p.(Leu513Met) and p.(Glu248Lys) in XYLT1. Desbuquois dysplasia type 2 has vast symptoms and complications. Therefore, due to the variety and severity of complications, identifying and reporting related mutations and using them in prenatal diagnosis is a useful diagnostic tool in the prevention of this disease and benefits children and families affected by this disease.

\section{Abbreviations \\ XT1: Xylosyltransferase 1; PG: Proteoglycan; GAG: Glycosaminoglycan; UDP- xylose: Uridine diphosphate-xylose; ECM: Extracellular matrix; WES: Whole- exome sequencing; HC: Head circumference; AC: Abdominal circumference; CD-PGs: Chondroitin sulfate proteoglycans; CS: Chondroitin sulfate; DS: Dermatan sulfate; HS: Heparan sulfate; ER: Endoplasmic reticulum; DBQD: Desbuquois dysplasia; NT: Nuchal translucency; FHR: Fetal heart rate.}

\section{Supplementary Information}

The online version contains supplementary material available at https://doi. org/10.1186/s12887-022-03132-5.

Additional file 1.

Acknowledgements

The Authors thank the family involved in this research.

\section{Authors' contributions}

MG performed the study. AHB collected data. FR and AHB performed mutation analysis. FR and MR wrote the draft manuscript. All authors have reviewed the manuscript and approved the content for publication.

\section{Funding}

None.

\section{Availability of data and materials}

All data generated or analyzed during this study are included in this published article.

Data sharing does not apply to this report as no data sets were generated or analyzed.

\section{Declarations}

Ethics approval and consent to participate

Ethics approval was not applicable. Written informed consent of the genetic testing was obtained from the patient's parents.

\section{Consent for publication}

Written informed consent for publication was obtained from patient's parents, also the individual information of the family members of this case report.

\section{Competing interests}

The authors declare no conflict of interest.

\section{Author details}

${ }^{1}$ Department of Medical Genetics, Faculty of Medical Sciences, Tarbiat Modares University, Tehran, Iran. ${ }^{2}$ Prenatal Diagnosis and Genetic Research Center, Dastgheib Hospital, Shiraz University of Medical Sciences, Shiraz, Iran. ${ }^{3}$ Department of Genetics and Molecular Medicine, Faculty of Medicine, Bushehr University of Medical Sciences, Bushehr, Iran.

Received: 15 May 2021 Accepted: 15 January 2022

Published online: 26 January 2022 


\section{References}

1. Jamsheer A, Olech EM, Kozłowski K, Niedziela M, Sowińska-Seidler A, Obara-Moszyńska M, et al. Exome sequencing reveals two novel compound heterozygous XYLT1 mutations in a Polish patient with Desbuquois dysplasia type 2 and growth hormone deficiency. J Human Genetics. 2016;61(7):577-83.

2. Bui C, Huber C, Tuysuz B, Alanay Y, Bole-Feysot C, Leroy JG, et al. XYLT1 mutations in Desbuquois dysplasia type 2. Am J Human Genet. 2014:94(3):405-14.

3. Kim OH, Nishimura G, Song HR, Matsui Y, Sakazume S, Yamada M, et al. A variant of Desbuquois dysplasia characterized by advanced carpal bone age, short metacarpals, and elongated phalanges: report of seven cases. Am J Med Genet Part A. 2010;152(4):875-85.

4. Inoue S, Ishii A, Shirotani G, Tsutsumi M, Ohta E, Nakamura M, et al. Case of $D$ esbuquois dysplasia type 1: Potentially lethal skeletal dysplasia. Pediatr Int. 2014;56(4):e26-e9.

5. Huber C, Oulès B, Bertoli M, Chami M, Fradin M, Alanay Y, et al. Identification of CANT1 mutations in Desbuquois dysplasia. The American Journal of Human Genetics. 2009;85(5):706-10.

6. van Koningsbruggen $\mathrm{S}$, Knoester $\mathrm{H}$, Bakx R, Mook O, Knegt L, Cobben JM. Complete and partial XYLT1 deletion in a patient with neonatal short limb skeletal dysplasia. American Journal of Medical Genetics Part A. 2016;170(2):510-4.

7. Al-Jezawi NK, Ali BR, Al-Gazali L. Endoplasmic reticulum retention of xylosyltransferase 1 (XYLT1) mutants underlying Desbuquois dysplasia type II. American Journal of Medical Genetics Part A. 2017;173(7):1773-81.

8. Schön S, Huep G, Prante C, Müller S, Christ R, Hagena F-W, et al. Mutational and functional analyses of xylosyltransferases and their implication in osteoarthritis. Osteoarthritis cartilage. 2006;14(5):442-8.

9. Heidari E, Caddeo A, Zarabadi K, Masoudi M, Tavasoli AR, Romeo S, et al. Identification of novel loss of function variants in MBOAT7 resulting in intellectual disability. Genomics. 2020;112(6):4072-7.

10. Lill MA, Danielson ML. Computer-aided drug design platform using PyMOL. J Comput Aided Mol Des. 2011;25(1):13-9.

11. Rodrigues $\mathrm{CH}$, Pires DE, Ascher DB. DynaMut: predicting the impact of mutations on protein conformation, flexibility and stability. Nucleic acids research. 2018;46(W1):W350-W5.

12. Viklund $H$, Elofsson A. OCTOPUS: improving topology prediction by two-track ANN-based preference scores and an extended topological grammar. Bioinformatics. 2008;24(15):1662-8.

13. Chaudhary P, Naganathan AN, Gromiha MM. Folding RaCe: a robust method for predicting changes in protein folding rates upon point mutations. Bioinformatics. 2015;31(13):2091-7.

14. Ranza E, Huber C, Levin N, Baujat G, Bole-Feysot C, Nitschke P, et al. Chondrodysplasia with multiple dislocations: comprehensive study of a series of 30 cases. Clin Genet. 2017:91(6):868-80.

15. Al-Dewik N, Mohd H, Al-Mureikhi M, Ali R, Al-Mesaifri F, Mahmoud L, et al. Clinical exome sequencing in 509 Middle Eastern families with suspected Mendelian diseases: The Qatari experience. American Journal of Medical Genetics Part A. 2019;179(6):927-35.

16. Guo L, Elcioglu NH, lida A, Demirkol YK, Aras S, Matsumoto N, et al. Novel and recurrent XYLT1 mutations in two Turkish families with Desbuquois dysplasia, type 2. Journal of human genetics. 2017;62(3):447-51.

17. Köhler S, Doelken SC, Mungall CJ, Bauer S, Firth HV, Bailleul-Forestier I, et al. The Human Phenotype Ontology project: linking molecular biology and disease through phenotype data. Nucleic acids research. 2014:42(D1):D966-D74.

18. Powis Z, Hagman KDF, Speare V, Cain T, Blanco K, Mowlavi LS, et al. Exome sequencing in neonates: diagnostic rates, characteristics, and time to diagnosis. Genet Sci. 2018;20(11):1468-71.

19. Briggs DC, Hohenester E. Structural basis for the initiation of glycosaminoglycan biosynthesis by human xylosyltransferase 1. Structure 2018;26(6):801-9. e3.

20. Götting C, Kuhn J, Zahn R, Brinkmann T, Kleesiek K. Molecular cloning and expression of human UDP-D-xylose: proteoglycan core protein $\beta$-D xylosyltransferase and its first isoform XT-II. Journal of molecular biology. 2000;304(4):517-28.

21. Munns CF, Fahiminiya S, Poudel N, Munteanu MC, Majewski J, Sillence DO, et al. Homozygosity for frameshift mutations in XYLT2 result in a spondylo-ocular syndrome with bone fragility, cataracts, and hearing defects. The American Journal of Human Genetics. 2015;96(6):971-8.
22. Schreml J, Durmaz B, Cogulu O, Keupp K, Beleggia F, Pohl E, et al. The missing "link": an autosomal recessive short stature syndrome caused by a hypofunctional XYLT1 mutation. Human genetics. 2014;133(1):29-39.

23. Mizumoto S, Yamada S, Sugahara K. Mutations in biosynthetic enzymes for the protein linker region of chondroitin/dermatan/heparan sulfate cause skeletal and skin dysplasias. BioMed Research International; 2015.

\section{Publisher's Note}

Springer Nature remains neutral with regard to jurisdictional claims in published maps and institutional affiliations.
Ready to submit your research? Choose BMC and benefit from:

- fast, convenient online submission

- thorough peer review by experienced researchers in your field

- rapid publication on acceptance

- support for research data, including large and complex data types

- gold Open Access which fosters wider collaboration and increased citations

- maximum visibility for your research: over $100 \mathrm{M}$ website views per year

At BMC, research is always in progress.

Learn more biomedcentral.com/submissions 